\title{
THE OCCURRENCE OF JAUNDICE IN THERAPEUTIC AND NATURAL MALARIA ${ }^{1}$ \\ BY THOMAS C. CHALMERS, JR. \\ (From the Department of Medicine, New York University and the Goldwater Memorial Hospital, New York City)
}

(Received for publication April 1, 1947)

The resurgence of interest in malaria occasioned by the war has resulted in several evaluations of damage to the liver by that disease. Two general types of material have been the basis for the studies: therapeutic malaria induced by the transfer of infected blood, and naturally acquired, mosquito-induced malaria. The differences between these 2 types as regards the characteristics of the host and the disease itself have not been taken sufficiently into account in the published work to date. The data in the following report indicate that these factors are important in an evaluation of the deleterious effect of malaria upon the liver.

Kopp and Solomon (1) followed 9 patients during fever therapy and found moderate changes in a number of liver function tests and frank jaundice in 1 subject. They also noted that jaundice occasionally appears after malaria has been terminated and arsphenamine treatment has been begun. Fredericks and Hoffbauer (2) found that all of 31 patients studied showed from slight to moderate changes in from 1 to all of the liver function tests employed. One patient developed jaundice 43 days after fever therapy and died of hepatic insufficiency. Guttman et al (3) reported functional changes in all of 6 patients studied, 1 of whom developed clinical jaundice. More recently, Read et al (4) reported an incidence for jaundice of 7.3 per cent in a group of 300 patients treated with malaria. None developed jaundice after the termination of their malaria, but 2 were inoculated from a third who later developed jaundice. All of these reports dealt with the blood-induced type of malarial infection.

Four studies of naturally occurring malaria have appeared more recently. Mirsky et al (5)

1 The work described in this paper was done in part under a contract, recommended by the Committee on Medical Research, between the Office of Scientific Research and Development and the New York University College of Medicine. studied 8 patients during malarial relapses. Four had icteric indices above 10 (highest 18), all developed a positive cephalin flocculation, and all had slightly elevated serum globulins. Kern and Norris (6) were impressed by the high incidence of enlarged liver in patients with acute and recurrent malaria seen in the Pacific Theatre of Operations. Jaundice was usually absent and when present was slight. In 2 of 18 patients the bromsulfalein retention was over 15 per cent in 30 minutes. Lippincott et al (7) performed many liver function studies on 317 soldiers with relapsing malaria over periods ranging up to 6 months. In general the changes found during an acute attack were much less striking than those reported for therapeutic malaria, and there was little or no evidence of liver damage. Finally, Tumulty et al (8) in a study of the effects of recurrent benign tertian malaria on the individual as a whole found no abnormalities in liver function as measured by the icteric index, and serum albumin and globulin in a total of 50 soldiers studied. The bromsulfalein excretion in 40 and the hippuric acid test in 10 , were also normal.

The following is a report on the incidence and character of hepatitis observed during the malarial treatment of central nervous system syphilis on the New York University Research Service of the Goldwater Memorial Hospital, New York City. The purpose of the investigations was to quantitate the efficacy of old and new antimalarial drugs and the observations of hepatitis reported in this paper are incidental. From 1942 through 1944, 450 patients received malarial therapy induced by blood inoculation. Of these, 36 , or 8 per cent, developed jaundice. In addition, 27 conscientious objectors and 7 syphilitics at Goldwater, and 65 volunteers at the Army Detention Barracks, Greenhaven, New York, were given the Chesson, or South Pacific, strain of vivax malaria 
by mosquito inoculation without the development of clinical jaundice. ${ }^{2}$

\section{MATERIALS AND METHODS}

The patients in the blood inoculation group were given initially either the $\mathrm{McCl}$ oy strain of $P$. vivax or the McLendon strain of $P$. falciparum. After 3 to 6 days of fever they received an antimalarial drug under study. If there was complete suppression of parasites for 2 or 3 weeks, they were then reinoculated from another donor and the second course of fever was allowed to run to a spontaneous termination. If this did not give them the required 75 hours of fever over $103^{\circ}$, they were inoculated with $P$. malariae or given a course of triple typhoid vaccine infusions. Thus most patients were inoculated more than once and their stay on the wards ranged from 1 to 6 months. Inoculations were performed by transferring from 0.1 to $4.0 \mathrm{cc}$. of citrated blood directly from one patient to the other, the amount depending on the parasite density of the donor's blood.

Some characteristics of the patients treated are important in evaluating the results. They were almost all referred by the Social Hygiene Clinics of the New York City Board of Health. In general they were from an economic stratum that is more prone to dietary deficiencies and alcoholism than the average civilian or soldier with naturally occurring malaria. In addition about 50 per cent had received fairly extensive arsenical treatment; only 11 per cent had received no prior antiluetic treatment.

The bromsulfalein excretion was examined routinely on admission so that observations are available on all but a few patients. The $5 \mathrm{mgm}$. per $\mathrm{kgm}$. dose was used. Any retention of over 15 per cent in 30 minutes, with a 5-minute specimen designated as 100 per cent, was considered abnormal. Of 400 patients on whom the test was done before treatment only 72 per cent were normal by these criteria. By contrast, all but 1 of the 27 conscientious objectors had readings well under 15 per cent, and the 1 exception had 2 determinations of 14 and 18 .

\section{RESULTS}

The only significant difference in history and preliminary work-up between patients who later developed jaundice and those who did not was in bromsulfalein retention. As shown in Table I, the former group had a significantly higher incidence of liver impairment demonstrated by that test than 100 patients picked at random from among those who did not develop jaundice. Differences were observed in the amount of previous exposure to the arsphenamines and excessive indulgence in alcohol, but these are not statistically

2 The author wishes to thank Dr. David P. Earle and Dr. Gordon Zubrod for supplying the data on these patients. significant. The age and sex range of the jaundiced patients was the same as that of the group as a whole. There was a somewhat higher incidence in those of the white race, but some attacks of jaundice may well have been missed in the negroes. No strain of malaria was associated more often with jaundice than any other.

Half of the 36 patients became jaundiced while they were febrile with malaria. These will be referred to hereafter as Group 1. Those occurring in the post-febrile period will be designated as Group 2. Of the 18 patients in Group 1, 6 occurred during the first bout of fever, 11 during the second and 1 during the third. In Group 2, 4 occurred after the first bout, 12 after the second and 2 after the third.

Fifty per cent of the patients in Group 1 and 88 per cent of these in Group 2 had either mild symptoms or none at all. The remainder were more severely ill with such symptoms as marked anorexia, vomiting, diarrhea, epigastric pain, pruritis, and in 1 case hematemesis and ascites. None died. Almost all had enlargement of the liver. The average duration of jaundice in Group 1 was 13 days and in Group 2, 7 days. The diagnosis of hepatitis was confirmed by tests for urinary bile and urobilinogen, icteric index or serum bilirubin and bromsulfalein excretion. In Group 1, 7 patients had no drop in hemoglobin over the

TABLE I

Incidence of abnormal bromsulfalein retention among patients prior to blood inoculation to establish malaria

21 patients who developed jaundice compared with 100 patients picked at random who did not develop jaundice.

\begin{tabular}{|c|c|c|c|c|c|}
\hline & \multicolumn{5}{|c|}{$\begin{array}{l}\text { Per cent bromsulfalein retention } \\
\text { in } 30 \text { minutes }\end{array}$} \\
\hline & $<16 \%$ & $<21 \%$ & $<31 \%$ & $<41 \%$ & $<51 \%$ \\
\hline Patients with jaun- & 12 & 15 & 18 & 20 & 21 \\
\hline $\begin{array}{l}\text { Patients with jaun- } \\
\text { dice, per cent }\end{array}$ & $57 \%$ & $71 \%$ & $86 \%$ & $95 \%$ & $100 \%$ \\
\hline $\begin{array}{l}\text { Patients without jaun- } \\
\text { dice, number and per } \\
\text { cent }\end{array}$ & 82 & 90 & 99 & 100 & \\
\hline
\end{tabular}

7 to 10 day period prior to the onset of jaundice, and 11 had an average drop of 1.3 grams, no more than the average patient has with the same amount of malaria. 


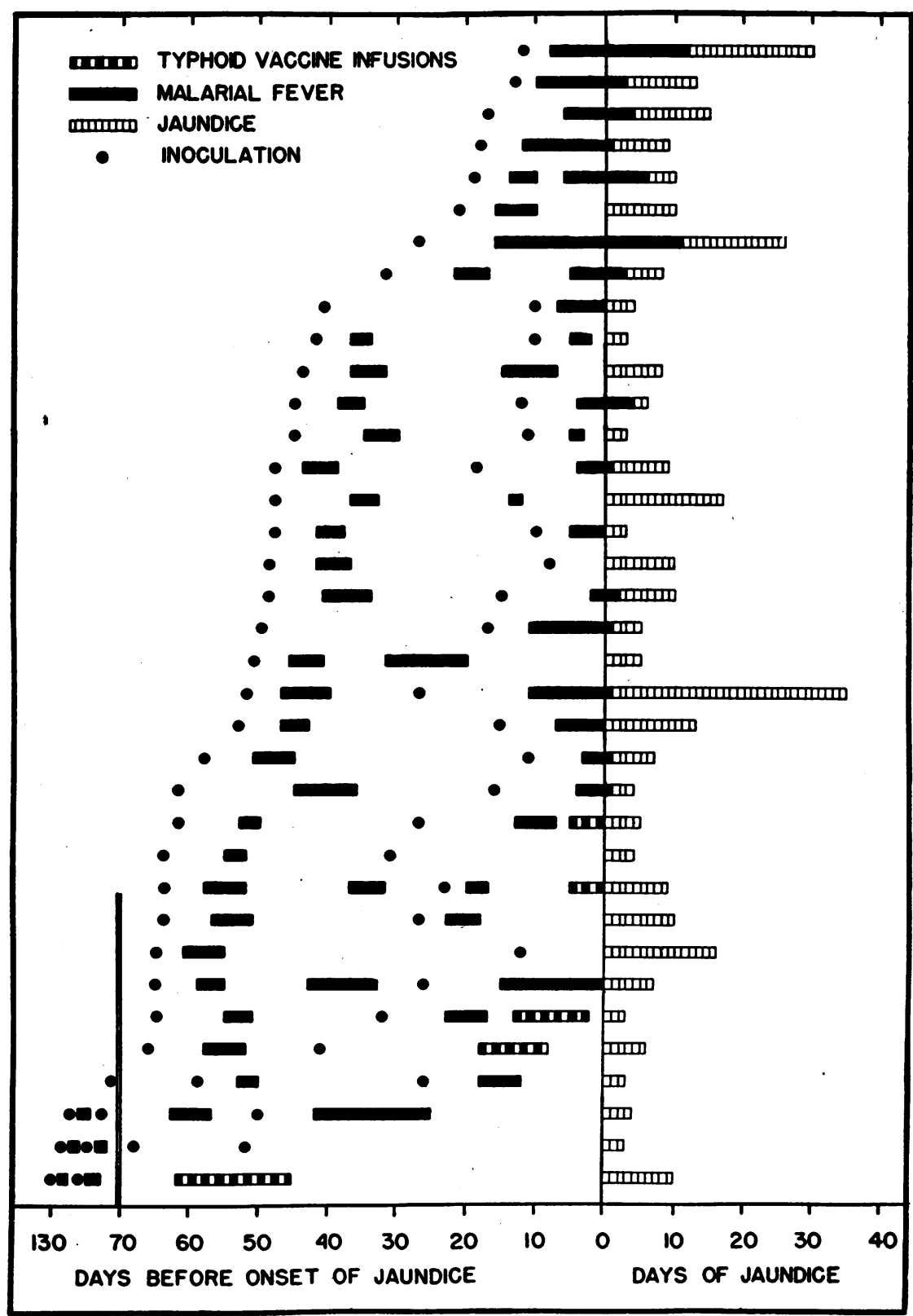

Fig. 1. Schematic Representation of the Time Relationships of the Inocelations with Blood to Induce Malaria, the Duration of Malarial and Typhoid Vaccine Fever, and the Onset and Duration of Jaundice

The day of onset of the jaundice occurs at the zero line; the scale changes at the far left.

The relationship between the time of inoculation and the onset of jaundice is presented in Figure 1. These data are presented in detail to illustrate the possibility of a transfer of an icterogenic virus with the transfer of blood utilized for inoculation of patients with malaria. In Table II the figures are broken down into groups and reveal that those who developed jaundice during malaria had in general shorter "incubation periods" than those who developed their jaundice in a malaria-free 
TABLE II

Number of days between inoculation and onset of jaundice (taking the maximum number of days when 2 or more inoculations were performed)

Group 1 includes those who developed jaundice while febrile with malaria, Group 2 those who developed it after their malaria had been terminated.

$\begin{array}{ccc}\text { Days } & \text { Group 1 } & \text { Group 2 } \\ 0-40 & 7 & 1 \\ 41-55 & 8 & 6 \\ 56-70 & 3 & 7 \\ 71-130 & 0 & 4\end{array}$

interval. In 28 of the patients, 78 per cent, the period between the first inoculation and onset of the jaundice was over 40 days. All patients were on the wards at least 3 weeks before the onset of jaundice.

There is additional data suggesting the possibility of transfer of an icterogenic agent in inoculation malaria. In 2 instances jaundice developed in each of 3 patients who had received their infected blood from a single donor, and there were 4 patients who donated blood to 2 others who later developed jaundice. The intervals in the former group were 12, 32 and 111 days in one instance, and 17, 26 and 42 days in the other. In the latter group the intervals were 8 and 16, 10 and 51,44 and 118, and 53 and 66 days. The records available do not indicate how many patients received blood from these same donors and did not develop jaundice.

\section{DISCUSSION}

As far as is known, none of the drugs used in these studies to cure the malaria is hepatotoxic in the doses administered. In only 1 of the patients could hepatitis possibly be related to a drug, and that was a sulfonamide which was given to other patients who did not develop hepatitis. There is no reason to believe that the typhoid vaccine infusions caused the jaundice. Some of the cases may have been sporadic infectious hepatitis. There were always 15 to 30 patients in various stages of treatment associating closely with each other on the male ward. The cases were evenly distributed over a $2 \frac{1}{2}$-year period. One nurse developed typical acute hepatitis during this period without known contact outside the hospital.

Since no attempt was made to prove the transmission of a virus in any of the above reported patients, the evidence for such an etiology of some of the jaundice occurring during and after therapeutic malaria is only suggestive. But the fact that in half of the patients hepatitis occurred after the termination of malaria suggests that some of that occurring during malaria may be of the same etiology.

The incubation periods are on the whole considerably shorter than those reported for homologous serum jaundice. Incubation periods as short as 2 weeks, however, have been reported (9). Neefe et al (10) have reported changes in liver function tests in from 12 to 35 days after inoculation, although jaundice did not appear until considerably later. It is possible that the combination of an arsphenamine-injured liver and an intervening debilitating disease such as malaria may considerably shorten the time ordinarily required for the development of jaundice.

Of great importance with regard to this work are the recent reports by Neefe et al (11) and by Havens (12) that the parenteral transfer of a virus isolated from an epidemic of infectious hepatitis was followed by the same incubation period as the oral transfer, 20 to 37 days. Their data strongly suggest that there are at least 2 strains of hepatitis virus, and it is possible that strains with short as well as long incubation periods may have been responsible for our cases.

The occasional occurrence of jaundice, after arsphenamines have been resumed following malaria, may well be homologous serum jaundice rather than the toxic effect of arsphenamines on top of that of malaria as suggested by Kopp and Solomon (1). Indeed, there may be a distinct similarity between the problems of arsphenamine and malaria hepatitis in luetic patients. The former has recently been shown to be caused largely by transmission of a virus through improperly sterilized equipment $(13,14)$, a plausible explanation for the increased incidence of arsphenamine hepatitis during epidemics of infectious hepatitis.

Since the disease may be extremely serious, and since immune globulin has been shown to prevent infectious hepatitis if given early enough (15, $16)$, it might be wise to give immune globulin to each patient inoculated by transfer of infected blood. The evidence for its effectiveness in preventing post-transfusion hepatitis is not as strik- 
ing, but it was successful in a study reported by Grossman et al (17).

The data presented suggest that care should be exercised in drawing conclusions about the effect of naturally occurring malaria on the liver from studies of blood inoculation malaria in syphilitic patients. The returned troops with relapsing malaria have, on the whole, more normal livers to start with, they are inoculated by mosquitoes rather than the transfer of human blood, and their malaria is usually treated promptly rather than allowed to run for 1 to 2 weeks.

\section{SUMMARY}

Thirty-six instances of jaundice complicated the treatment of 450 syphilitics with malarial fever induced by blood inoculation. Only half of these occurred during an actual attack of malaria.

None of 99 mosquito-inoculated patients developed jaundice.

The possibility that some of these attacks of hepatitis were caused by a specific icterogenic virus is discussed.

Evidence is presented to show that an appreciable number of patients with central nervous system syphilis have impaired hepatic function before they are given malaria.

\section{ACKNOWLEDGMENT}

The author is indebted to Dr. James A. Shannon, former Director of the New York University Research Unit, Goldwater Memorial Hospital, for guidance in the preparation of this paper.

\section{BIBLIOGRAPHY}

1. Kopp, I., and Solomon, H., Liver function in therapeutic malaria. Am. J. M. Sc., 1943, 205, 90.

2. Fredericks, M. G., and Hoffbauer, F. W., A study of hepatic function in therapeutic malaria. J. A. M. A., 1945, 128, 495.

3. Guttman, S. A., Potter, H. R., Hanger, F. M., Moore, D. B., Pierson, P. S., and Moore, D. H., Signifi- cance of cephalin-cholesterol flocculation test in malarial fever. J. Clin. Invest., 1945, 24, 296.

4. Read, H. S., Kaplan, L. I., Becker, F. T., and Boyd, M. F., An analysis of complications encountered during therapeutic malaria. Ann. Int. Med., 1946, 24, 444.

5. Mirsky, I. A., von Brecht, R., and Williams, L. D., Hepatic dysfunction in malaria. Science, 1944, 99, 20.

6. Kern, R. A., and Norris, R. F., Liver involvement in malaria. U. S. Nav. Med. Bull., 1944, 43, 847.

7. Lippincott, S. W., Ellerbrook, L. D., Hesselbrock, W. B., Gordon, H. H., Gottlieb, L., and Marble, $\mathrm{H}$., Liver function tests in chronic relapsing vivax malaria. J. Clin. Invest., 1945, 24, 616.

8. Tumulty, P. A., Nichols, E., Singewald, M. L., and Lidz, T., An investigation of the effects of recurrent malaria; organic and psychological analysis of 50 soldiers. Medicine, 1946, 25, 17.

9. Neefe, J. R., Miller, T. B., and Chornock, F. W., Homologous serum jaundice. A review of the literature and report of a case. Am. J. M. Sc., 1944, 207, 626.

10. Neefe, J. R., Stokes, J. Jr., Reinhold, J. G., and Lukens, F. D. W., Hepatitis due to the injection of homologous blood products in human volunteers. J. Clin. Invest., 1944, 23, 836.

11. Neefe, J. R., Gellis, S. S., and Stokes, J., Jr., Homologous serum hepatitis and infectious (epidemic) hepatitis. Am. J. of Med., 1946, 1, 3.

12. Havens, W. P., Jr., Experiment in cross immunity between infectious hepatitis and homologous serum jaundice. Proc. Soc. Exp. Biol. \& Med., 1945, 59, 148.

13. Solomon, M. H., King, A. J., Williams, D. I., and Nicol, C. S., Prevention of jaundice resulting from anti-syphilitic treatment. Lancet, 1944, 2, 7.

14. Sheehan, H. L., Epidemiology of infectious hepatitis. Lancet, 1944, 2, 8.

15. Stokes, J., Jr., and Neefe, J. R., The prevention and attenuation of infectious hepatitis by gamma globulin. J. A. M. A., 1945, 127, 144.

16. Havens, W. P., and Paul, J. R., Prevention of infectious hepatitis with gamma globulin. J. A. M. A., 1945, 129, 270.

17. Grossman, E. B., Stewart, S. G., and Stokes, J., Jr., Post-transfusion hepatitis in battle casualties and study of its prophylaxis by means of human immune serum globulin. J. A. M. A., 1945, 129, 991. 